\title{
Matter-wave dark solitons in boxlike traps
}

\author{
M. Sciacca* \\ Dipartimento Scienze Agrarie e Forestali, Università di Palermo, Viale delle Scienze, Palermo 90128, Italy \\ and Istituto Nazionale di Alta Matematica, Roma 00185, Italy \\ C. F. Barenghi and N. G. Parker \\ Joint Quantum Centre Durham-Newcastle, School of Mathematics and Statistics, Newcastle University, \\ Newcastle upon Tyne, NE1 7RU, United Kingdom \\ (Received 28 September 2016; revised manuscript received 6 December 2016; published 25 January 2017)
}

\begin{abstract}
Motivated by the experimental development of quasihomogeneous Bose-Einstein condensates confined in boxlike traps, we study numerically the dynamics of dark solitons in such traps at zero temperature. We consider the cases where the side walls of the box potential rise either as a power law or a Gaussian. While the soliton propagates through the homogeneous interior of the box without dissipation, it typically dissipates energy during a reflection from a wall through the emission of sound waves, causing a slight increase in the soliton's speed. We characterize this energy loss as a function of the wall parameters. Moreover, over multiple oscillations and reflections in the boxlike trap, the energy loss and speed increase of the soliton can be significant, although the decay eventually becomes stabilized when the soliton equilibrates with the ambient sound field.
\end{abstract}

DOI: 10.1103/PhysRevA.95.013628

\section{INTRODUCTION}

Dark solitons are one-dimensional nondispersive waves which arise in defocusing nonlinear systems as localized depletions of the field envelope [1]. To date, they have been observed in systems ranging from optical fibers [2-4], magnetic films [5], plasmas [6], and waveguide arrays [7] to water [8] and atomic Bose-Einstein condensates [9]. This work is concerned with the last system; here the matter field of the gas experiences a defocusing cubic nonlinearity arising from the repulsive short-range atomic interactions. In the limit of zero temperature, the mean matter field is governed by a cubic nonlinear Schrödinger equation (NLSE) called the GrossPitaevskii equation (GPE) [10-14]. Many experiments have generated and probed these matter-wave dark solitons [15-24].

A necessary feature of an atomic condensate is the trapping potential required to confine it in space. When the trapping potential is highly elongated in one direction compared to the other two, the condensate becomes effectively one dimensional, and its longitudinal dynamics is described by the onedimensional (1D) GPE. If the system is homogeneous in the longitudinal direction, the GPE is integrable and supports exact dark soliton solutions. Dark solitons appear as a local notch in the atomic density with a phase slip across it, and travel with constant speed $[9,25]$ while retaining their shape. However, the presence of confinement in the longitudinal direction breaks the complete integrability of the governing equation and causes the dark soliton to decay via the emission of sound waves [26-30]. An analogous effect arises in nonlinear optics due to inhomogeneities of the optical nonlinearity $[1,31]$. In

\footnotetext{
*Corresponding author: michele.sciacca@unipa.it

Published by the American Physical Society under the terms of the Creative Commons Attribution 4.0 International license. Further distribution of this work must maintain attribution to the author(s) and the published article's title, journal citation, and DOI.
}

condensates, dark solitons may also decay through thermal dissipation [32-34] and transverse snaking instability into vortex pairs or rings [17,35-40]; both decay channels can be effectively eliminated by operating at ultracold temperatures and in tight 1D geometries, respectively.

To date, the trapping potentials most commonly used have been harmonic (quadratic in the distance from the center of the condensate). Evolution and stability of dark solitons moving under a longitudinal harmonic potential have been carefully analyzed. We know that the soliton tends to oscillate back and forth through the condensate at a fixed proportion of the trap's frequency [26,27,32,35,36]. While the inhomogeneous potential leads to sound emission from the soliton, the harmonic trap uniquely supports an equilibrium between sound emission and reabsorption, such that the soliton decay is stabilized [28,30]. Theoretical work has also considered the radiative behavior of a dark soliton moving under the effect of linear potentials and steps [41], perturbed harmonic traps [26,28,42], optical lattices [43,44], localized obstacles [41,45-50], anharmonic traps [30,47], and disordered potentials [51]. For slowly varying potentials, it is found that the power emitted by the solition is proportional to the square of the soliton's acceleration [1,28,31].

Increasingly, however, experiments are employing boxlike traps to produce quasihomogeneous condensates. Such traps have been realized in one [52,53], two [54], and three [55] dimensions [with tight harmonic trapping in the remaining directions in the 1D and two-dimensional (2D) cases]. These new traps feature flat-bottomed central regions and end-cap potential provided by optical or electromagnetic fields; the boundaries are therefore soft, unlike infinite hard walls of existing mathematical models. For example, the 1D optical box trap of Ref. [52] featured approximately Gaussian walls, while the $2 \mathrm{D}$ and three-dimensional (3D) optical box traps of Refs. [54,55] had a power-law scaling in the range from $x^{10}$ to $x^{15}$. In the bulk of the box trap, where the density is homogeneous, a dark soliton is expected to propagate at constant speed and retain its shape; however, the nature of the 
reflection of the soliton from boundaries which are steeper than the traditional quadratic dependence and softer than hard boundaries is still unexplored. Here we seek to address this problem through a systematic computational study of the reflection of a dark soliton from power law and Gaussian walls.

\section{MATHEMATICAL MODEL}

We consider an atomic condensate in the limit of zero temperature, with arbitrary trapping $V(x)$ along the axis and tight harmonic trapping in the transverse directions of frequency $\omega_{\perp}$. Neglecting thermal and quantum fluctuations, the condensate is described on a mean-field level by the 3D Gross-Pitaevskii equation (GPE) [56]. This 3D equation can be mapped on to an effective $1 \mathrm{D}$ equation providing the following criteria are satisfied [57,58]. First, the transverse size of the condensate, most appropriately parametrized by the transverse harmonic length $\ell_{\perp}=\sqrt{\hbar / m \omega_{\perp}}$, is narrower than the $3 \mathrm{D}$ healing length $\xi_{3 \mathrm{D}}$, which characterizes the minimum length scale for density variations within the condensate. This condition strongly suppresses the excitation of transverse modes and is equivalent to requiring that the transverse trapping energy $\hbar \omega_{\perp}$ exceeds the $3 \mathrm{D}$ chemical potential $\mu_{3 \mathrm{D}}$, the eigenvalue of the 3D GPE. Second, the axial extent of the condensate should exceed $\xi_{3 \mathrm{D}}$ such that the excitation of axial modes is favoured. Under the first assumption, the condensate tends to the transverse ground harmonic oscillator state in the transverse plane, which is static; integrating this out leads to the effective 1D GPE. We assume the quasi-1D limit in this work.

We describe the condensate by the one-dimensional wave function, $\Psi(x, t)$; the atomic density follows as $n(x, t)=$ $|\Psi(x, t)|^{2}$. The dynamical evolution equation of $\Psi$ is governed by the one-dimensional GPE,

$$
i \hbar \Psi_{t}=-\frac{\hbar^{2}}{2 m} \Psi_{x x}+V(x) \Psi+g|\Psi|^{2} \Psi,
$$

where $m$ is the atomic mass and the effective 1D nonlinear coefficient $g=2 \hbar \omega_{\perp} a_{s}$ arises from short-range atomic interactions of $s$-wave scattering length $a_{s}$, and subscripts denote partial derivatives.

Since we are concerned with quasihomogeneous condensates, it is natural to adopt units relating to the bulk of the 1D condensate, where the density is $n_{0}=\sqrt{\mu_{0} / g}$ [62], and the chemical potential $\mu_{0}$ is the characteristic energy scale. The healing length $\xi_{0}=\hbar / \sqrt{m n_{0} g}$ is the minimum spatial scale of density variations, and the speed of sound $c=\sqrt{n_{0} g / m}$ is the typical speed scale; the natural time scale of the bulk condensate follows as $\xi_{0} / c_{0}$. Employing these quantities as units leads to the following dimensionless GPE [62],

$$
\mathrm{i} u_{t}=-\frac{1}{2} u_{x x}+|u|^{2} u+V(x) u,
$$

where all variables are in their dimensionless form. Throughout the rest of this paper we employ dimensionless variables.

The total energy of the condensate, given by the integral,

$$
E_{\mathrm{tot}}=\int\left(\frac{1}{2}\left|\psi_{x}\right|^{2}+V(x)|\psi|^{2}+\frac{1}{2}|\psi|^{4}\right) d x,
$$

is conserved within the GPE, as confirmed by our numerical simulations.
Equation (2.2) has been investigated over the years in terms of the complete integrability (see Ref. [59] and reference therein). This property (even though still not univocally defined) regards the existence of infinite number of conservation laws and the possibility of relating the nonlinear PDE (partial differential equation) to a linear PDE by an explicit transformation. The main feature is that Eq. (2.2) is not completely integrable except for the case $V(x)=a x+b$, with $a$ and $b$ constants [59]. For $V(x)=0$, the GPE (2.2) has the following exact dark soliton solution $[1,9]$,

$$
u=\left\{k \tanh \left[k\left(x-t v-s_{0}\right)\right]+\mathrm{i} v\right\} e^{-\mathrm{i}\left(t-\theta_{0}\right)},
$$

where $k=\sqrt{1-v^{2}}$ is the amplitude of the dark soliton, $v$ is the soliton's speed (and $|v|<1$ ), and $s_{0}$ and $\theta_{0}$ are arbitrary reference values of the position and phase of the soliton. The normalized dark soliton's energy is [1]

$$
E_{\mathrm{sol}}=\frac{4}{3}\left(1-v^{2}\right)^{3 / 2} \text {. }
$$

In the absence of the external potential $[V(x)=0]$, the soliton (2.4) propagates without any loss along the BEC. This lossless motion results from the perfect balance of nonlinear $\left(|u|^{2} u\right)$ and linear $\left(u_{x x}\right)$ terms in Eq. (2.2). As in optics, the soliton may be seen as the envelope of different plane waves (harmonics) with different frequencies and phase velocities which moves with the group speed $v$, and the two terms $\left(|u|^{2} u\right.$ and $\left.u_{x x}\right)$ induce self-phase modulation (SPM) and group velocity dispersion (GVD), respectively. When the balance between the two terms ceases or is altered, some harmonic components acquire more energy or new harmonics are generated by the nonlinearity, and what one sees is the generation of small-amplitude density (sound) waves (as also explained in Sec. IV).

Dark solitons are dimensionally stable in 1D. However, as the transverse confinement of the condensate is relaxed from the quasi-1D limit, this stability breaks down. The 3D dark soliton nodal plane becomes prone to a bending instability, the snake instability, which tears the dark soliton apart into vortex rings (or vortex-antivortex pairs in $2 \mathrm{D}$ geometries) [17,35-40,46]. This represents the fact that, as the transverse width is increased, vortex rings (pairs) become energetically favored $[60,61]$. This instability kicks in when the transverse size of the condensate becomes greater than approximately $10 \xi_{3 \mathrm{D}}$ [60].

Our work is based on numerical simulations of the dimensionless 1D GPE (2.2). Numerical time integration of the equation is performed using the split-step Fourier method. The initial condition consists of the ground-state condensate solution obtained via the technique of imaginarytime propagation of the GPE, into which a dark soliton solution of (2.4) is multiplied at the origin (this solution is appropriate because at the origin the system is locally homogeneous). During the course of the longest simulations (e.g., Fig. 6) the total energy of the system, $E_{\text {tot }}$, changes by less than 1 part in $10^{4}$.

We consider two types of quasihomogeneous box potentials. The first, termed the power-law box and motivated by the experiments of Refs. [54,55], is characterized by boundaries where the potential increases as a power of the 
(a)
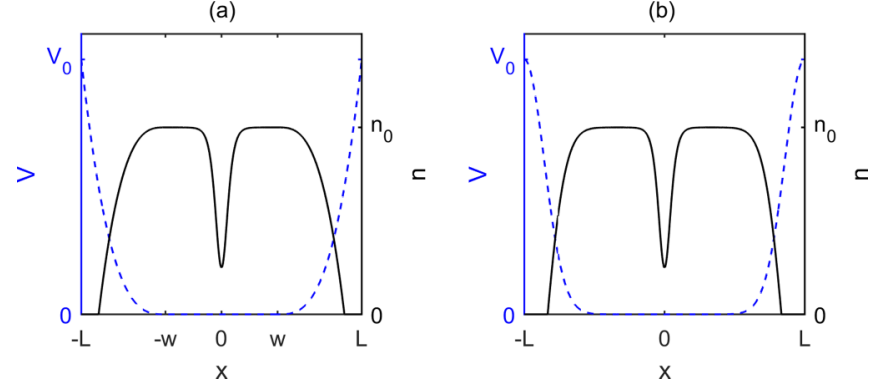

(a)
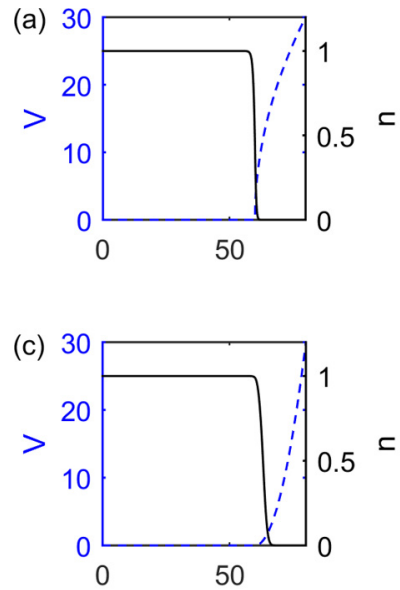

(e)

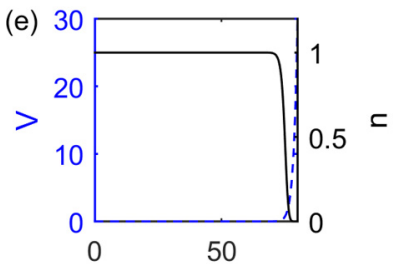

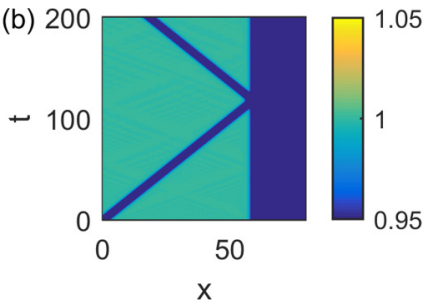

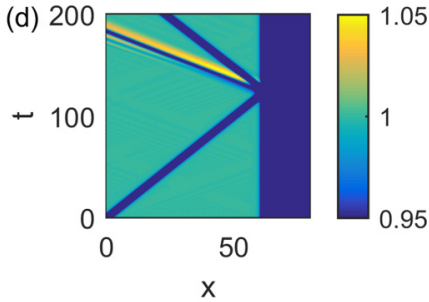
$V_{0}=V(L)$ at the edges $x= \pm L$ of the box, Gaussian potentials (centered at $x= \pm L$ and with width $L-w$ ) form the end caps of the box.

spatial coordinate. The overall potential has the form

$$
V(x)=\left\{\begin{array}{ll}
0 & \text { if }|x| \leqslant w \\
\left(\frac{|x|-w}{l}\right)^{\alpha} & \text { if } w<|x| \leqslant L
\end{array},\right.
$$

where $\alpha$ is the exponent of the potential at the boundaries, $2 w$ is the width of the flat part of the potential, and $2 L$ is the whole width of the potential. This potential is shown schematically in Fig. 1(a). The width of the boundary is then $L-w$. The height of the potential wall is given by $V_{0}=V(L)=\left(\frac{L-w}{l}\right)^{\alpha}$, and $l$ is a parameter used to establish the height of the side of the potential. The parameters we modify in our numerical experiments are the exponent $\alpha$, the height of the boundary potential $V_{0}$, and the width of the boundary potential $L-w$.

The second form of quasihomogeneous box trap is that where the end caps are formed by laser-induced Gaussian potentials, as used in Refs. [52]. This box, termed the exponential box and shown schematically in Fig. 1(b), has the form

$$
V(x)=V_{0}\left[e^{-\frac{(x-L)^{2}}{c^{2}}}+e^{-\frac{(x+L)^{2}}{c^{2}}}\right] .
$$

The crest of the Gaussian potentials are located at $x= \pm L, \quad V_{0}$ is their amplitude, and $c$ characterizes their width. As in the power-law box, we perform numerical experiments to explore the dependence on the amplitude $V_{0}$ and the width $c$ of the boundary potentials on the soliton's motion.

\section{RESULTS}

In Sec. III A we shall examine a single reflection of a dark soliton with a boundary of the box, for both the power-law and exponential box types. Later, in Sec. III B, we shall extend our analysis to multiple oscillations and reflections in the box. Throughout this section we set the box width to the arbitrary value $L=80$. (Units were defined above Eq. (2.2).)

\section{A. Single reflection}

A dark soliton (2.4) is introduced at the origin with arbitrary speed $v=0.5$ (in the positive $x$ direction) and launched at the $x=L$ boundary of the power-law box. Figure 2 shows the dynamics during the reflection from a power-law boundary

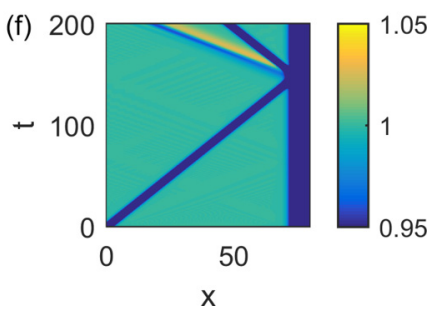

FIG. 2. Examples of the reflection of a $v=0.5$ dark soliton from the boundary of a power-law box. Shown are (a, c, e) the box potential (2.6) [left axes, dashed blue lines] and ground-state density profile [right axes, solid black lines], and (b, d, f) the evolution of the density during the reflection. Plots $(\mathrm{a}, \mathrm{b})$ correspond to a power-law exponent of $\alpha=0.5,(\mathrm{c}, \mathrm{d})$ correspond to $\alpha=2$, and (e, f) correspond to $\alpha=13$. The remaining parameters are fixed to $L=80, w=60$, and $V_{0}=30$.

with fixed width $w=60$ and amplitude $V_{0}=30$, and three different exponents. For $\alpha=0.5$ [Figs. 2(a) and 2(b)], the soliton reflects elastically. Here the boundary looks effectively like a hard wall: Up to the typical energy scale of the condensate, $V \sim 1$, the potential remains very steep. For a quadratic potential $\alpha=2$ [Figs. 2(c) and 2(d)], however, a pulse of sound waves is generated during the reflection which propagate in the negative $x$ direction at the speed of sound. These waves have amplitude of around $\sim 5 \%$ of the peak density. For a much higher exponent $\alpha=13$ [Figs. 2(e) and 2(f)], again sound waves are emitted during the reflection, with a slightly reduced amplitude.

Figure 3 shows example dynamics for the soliton reflecting from an exponential boundary. Here the amplitude of the boundaries is fixed to $V(L)=30$ and the width $c$ varied. For a narrow edge $c=1(\mathrm{a}, \mathrm{b})$ the soliton reflects elastically. Like above, the boundary appears as a hard wall. However, for wider boundaries, $c=4(\mathrm{c}, \mathrm{d})$ and $c=10(\mathrm{e}, \mathrm{f})$, the soliton dissipates energy through the emission of sound waves.

It is evident that the reflection of the dark soliton from the soft boundary is typically dissipative (where we are referring to the dissipation of the soliton; the total energy of the system is conserved), although the amount of sound radiated is sensitive to the boundary parameters. Now we characterize this dissipation in terms of the energy lost from the soliton. The soliton's energy $E_{\text {sol }}$ is evaluated numerically before 

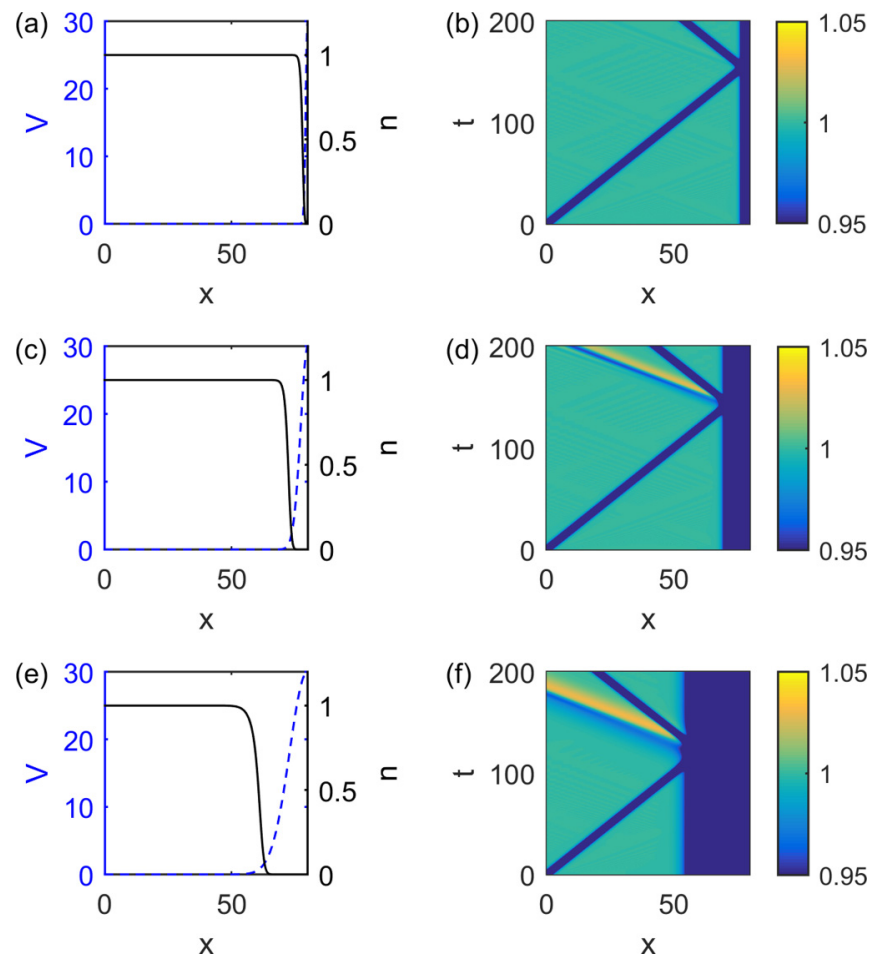

FIG. 3. Examples of the reflection of a $v=0.5$ dark soliton from the boundary of an exponential box. Shown are (a, c, e) the box potential (2.7) [left axes, dashed blue lines] and ground-state density profile [right axes, solid black lines], and (b, d, f) the evolution of the density during the reflection. Plots $(\mathrm{a}, \mathrm{b})$ correspond to a Gaussian width of $c=1,(\mathrm{c}, \mathrm{d})$ correspond to $c=4$, and (e, f) correspond to $c=10$. The remaining parameters are fixed to $L=80$ and $V_{0}=30$.

and after the reflection. This is performed by calculating the energy associated with the soliton within a small region around the soliton, according to the scheme described in Ref. [30]. We report the proportional loss in soliton energy after the reflection, normalized with respect to its initial value, and denote this as $\Delta E_{\mathrm{sol}}$.

Figure 4(a) shows the energy loss for the power-law trap as a function of the amplitude of the boundary potential $V_{0}$, for three values of the potential exponent $\alpha$. Note that we limit our analysis to $V_{0} \geqslant 2$; below this range the potential does not fully confine the condensate. For $\alpha=2$ and $\alpha=13$, the energy loss increases to a maximum at moderate $V_{0}\left(V_{0} \sim 5-10\right.$ for these cases), before decaying with increasing $V_{0}$. This is typical of the general behavior for $\alpha \geqslant 1$. It is worth noting that the softer boundary, $\alpha=2$, gives the most energy loss (up to 5\%), and that the energy loss decays very slowly with $V_{0}$, and so causes significant dissipation even for large amplitudes. For $\alpha<1$, however, the trend is distinct. For large $V_{0}$, sound emission is heavily suppressed; this is because for $\alpha<1$ the boundary potential rises up with a very large gradient (which decreases with distance into the boundary). As such, for $V_{0} \gg 1$ the condensate or soliton effectively experiences a hard wall potential. For smaller $V_{0}$, however, the condensate or soliton experiences the low-gradient region of the boundary, inducing sound emission. The energy loss increases rapidly as $V_{0}$ is decreased towards the value of 2 , enhanced by an unusual effect where sound waves are generated from the
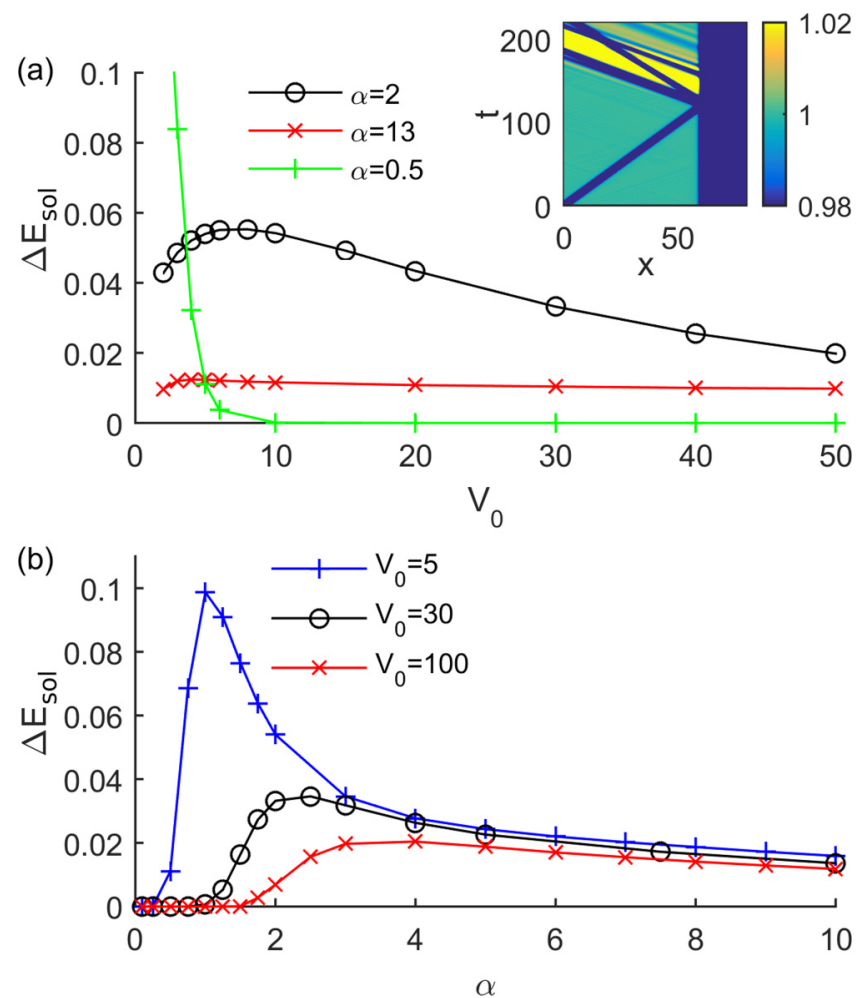

FIG. 4. Energy loss in the dark soliton (normalized by its initial energy) $\Delta E_{\text {sol }}$ due to a reflection against a power-law boundary. Panel (a) shows this energy loss as a function of the amplitude of the boundary potential $V(L)$, for three values of the exponent $\alpha$. Panel (b) shows $\Delta E_{\mathrm{sol}}$ as a function of the exponent $\alpha$ for fixed potential amplitude $V_{0}$. In panel (a) the inset is for $\alpha=0.5$ and $V_{0}=2$, showing anomalously high sound emission.

boundary even after the soliton has left the boundary [see inset of Fig. 4(a)].

Figure 4(b) shows the energy loss as a function of the exponent $\alpha$, for three values of the potential amplitude. The general behavior is that the energy loss is typically vanishingly small for small $\alpha$, due to the hard-wall effect mentioned above, and is also small for very large $\alpha$, since the potential increases rapidly and also begins to approximate a hard wall. However, in between these limits, the energy loss reaches maximum; the position of this maximum is dependent on $V_{0}$ but typically lies in the range $1<\alpha<5$.

Similarly, we have explored the energy loss from a single reflection of an exponential boundary. For fixed width $c$ [Fig. 5(a)], the energy loss is highest for the lowest amplitudes, and decreases as $V_{0}$ is increased. Meanwhile, for fixed amplitude $V_{0}$ [Fig. 5(b)] the energy loss is vanishingly small for small width $c$; here the exponential wall is so narrow that it resembles the hard wall. The energy loss increases with $c$, reaches a maximum for moderate values $c \sim 5-10$, and then decreases slowly with $c$. The energy loss is typically of the order of a few percent.

\section{B. Multiple reflections}

In a single reflection, the energy loss from the soliton is small, typically of the order of a few percent, and the increase 

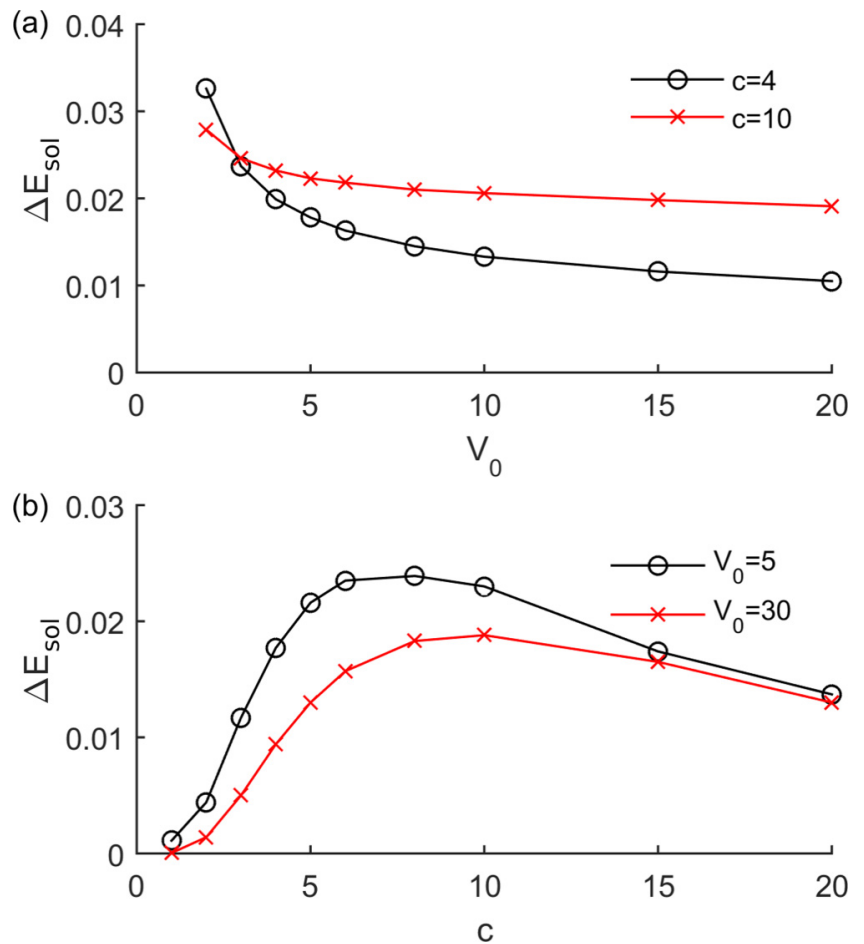

FIG. 5. Energy loss in the dark soliton (normalized by its initial energy) $\Delta E_{\text {sol }}$ due to a reflection against an exponential boundary. Panel (a) shows this energy loss as a function of the amplitude of the boundary potential $V_{0}$, for two values of the Gaussian width $c$, while panel (b) displays it as a function of the $c$ for fixed potential amplitude $V_{0}$.

in its speed is so small that it is not visible by eye. However, in the course of multiple reflections, such as due to a dark soliton oscillating back and forth in a box trap, significant decay of the soliton can be expected.

Figure 6 shows the long-term evolution of a dark soliton, with initial speed $v=0.5$, oscillating back and forth in a power-law trap (parameters $\alpha=2, V_{0}=5$ ). With each reflection the soliton loses amplitude and speeds up, while the condensate becomes increasingly populated with density waves. After of the order of 25 reflections the soliton has reached a speed $v \sim 0.9$. Interestingly, at late times (see upper plot), additional fast dark soliton-like structures (low density, localized structures) appear to pass back and forth through the box.

To quantify the decay of the soliton during the repeated oscillations through the box we monitor the speed of the soliton through the bulk of the condensate following each reflection. Figure 7(a) shows the soliton speed versus the number of reflections $N_{\mathrm{r}}$ for two power-law boxes, while Fig. 7(b) shows the corresponding behavior for the soliton's energy, calculated using the energy-speed relation for a dark soliton (2.5). The qualitative behavior is general: The soliton speed increases and relaxes towards a maximum value (which is less than unity), while the soliton's energy decays towards a value (which exceeds zero). The trends are captured by an exponential fit (solid lines). Importantly, these results shows that the soliton does not decay away completely, but saturates towards a high-speed and low-energy state. By these late times, the system is full of density waves of similar amplitude,
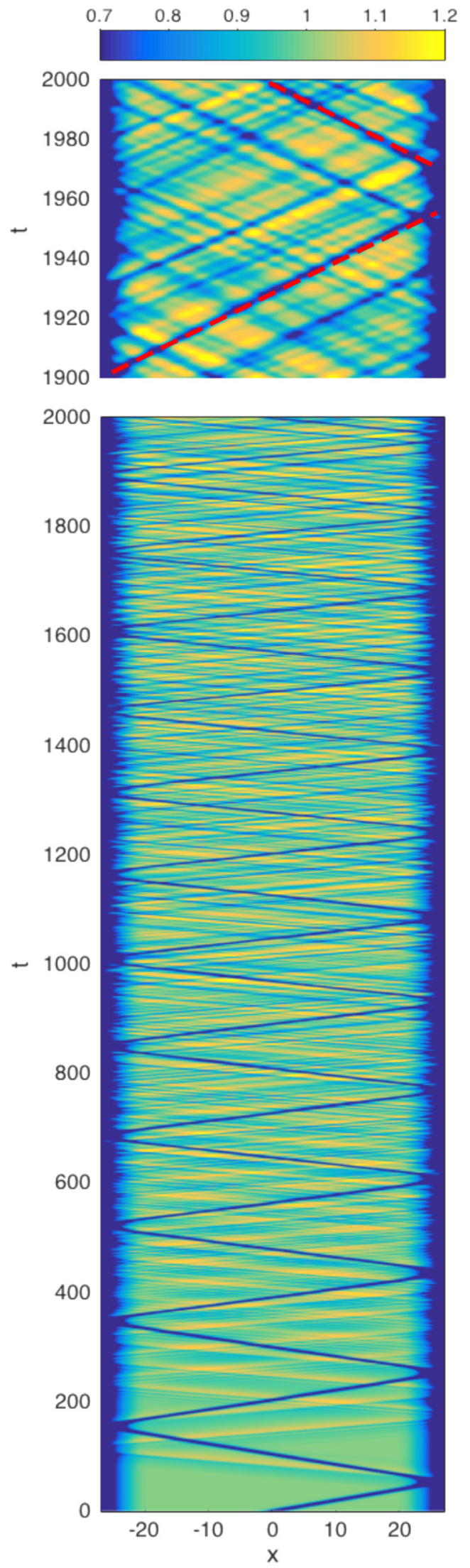

FIG. 6. Dark soliton (initial speed $v=0.5$ ) oscillating in a powerlaw box trap. The upper plot shows a closeup over the time range [1900, 2000], with the original solition indicated by the dashed red line. Trap parameters $V_{0}=5, \alpha=2, L=40$, and $w=20$. 

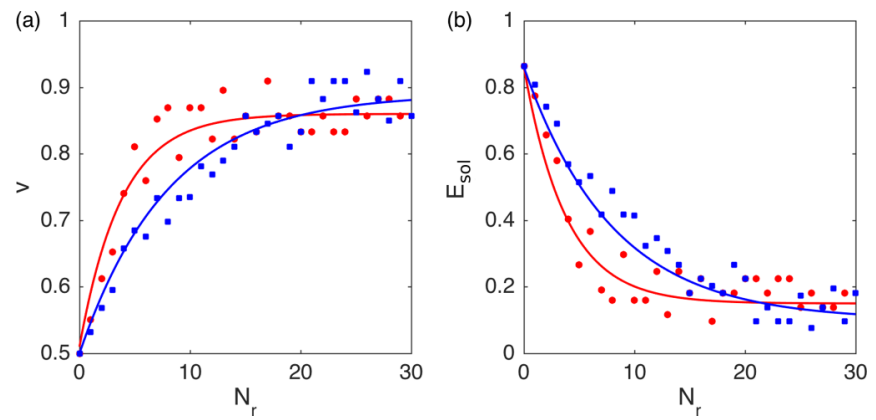

FIG. 7. Decay of a soliton with initial speed $v=0.5$ after multiple reflections in a power-law trap. (a) Soliton speed after $N_{\mathrm{r}}$ reflections for $\alpha=1$ (red circles) and $\alpha=2$ (blue squares). The speed is measured as the average speed through the bulk of the condensate. (b) The soliton's energy $E_{\text {sol }}$, determined using the speed-energy relation (2.5). In panels (a) and (b) the red and blue lines are exponential fits to the $\alpha=1$ and $\alpha=2$ data, respectively. Other parameters are $V_{0}=5, L=40$, and $w=20$.

suggesting that the decay may be stabilized by absorption of energy from the density waves.

\section{DISCUSSION}

We have seen that the reflection of the dark soliton from a soft wall is typically dissipative, in that the soliton loses energy through the emission of sound waves. In this section we try to understand what happens to the dark soliton when it interacts with the soft wall of the potential, where the balancing between the two terms in the Gross-Pitaevskii equation (2.2) (the linear term $u_{x x}$ and the nonlinear term $|u|^{2} u$ ) is altered by the presence of the potential term $V(x)$. This kind of problem is a classical problem in the context of complete integrability of a PDE, and similar problems have been also dealt with for optical fibers, which are both dispersive and dissipative. The parallels between optical fibers and 1D BECs may be useful for our purposes, supported also by the fact that the evolution equation of a dark solition in a normal-dispersion optical fiber, the nonlinear Schrödinger equation, is essentially the Gross-Pitaevskii equation (2.2) with time and spatial coordinates inverted.

In Fig. 6 the dissipation of the dark soliton during the multiple reflections in a power-law box trap is evident. The dissipation does not occur in the central, flat region of the condensate $(-w<x<w)$, where the soliton propagates undisturbed. This is the expected behavior of the soliton due to the integrability of the GPE (given that $V(x)=0$ for $x \in[-w, w]$ ). What happens at $x= \pm w$, i.e., when the dark soliton reaches the wall of the potential? We note that the soliton is made up of many harmonics (it is the envelope of the density waves), that the linear term $u_{x x}$ allows each harmonic to propagate with its own speed, while the nonlinear term $|u|^{2}$ is responsible for the generation of new harmonics and for continuously reorganizing the energy of the soliton over all the harmonics (supplying energy from some harmonics to others or to new harmonics) in such a way that the soliton propagates unchanged (as occurring in an optical fiber). At the wall, the nonlinear term $|u|^{2} u$ becomes modified to $\left[|u|^{2}+V(x)\right] u$ and its balance with the linear term $u_{x x}$ is modified. We claim, as shown below, that this mechanism is responsible for supplying energy to some harmonics which then escape from the soliton, making the potential wall of the trap dissipative for the dark soliton.

We consider the GPE (2.2) without the dispersion term $u_{x x}$ (which is responsible only for the dispersion mechanisms) in order to understand how the potential $V(x)$ modifies the generation of wave numbers. The solution of the GPE is then straightforward to find, taking the form $u(x, t)=$ $|u(x, 0)| \exp (\mathrm{i} \phi)$, where the phase $\phi=\left[|u(x, 0)|^{2}+V(x)\right] t$ depends on $x, t$, and the instantaneous wave number $\mathrm{K}=$

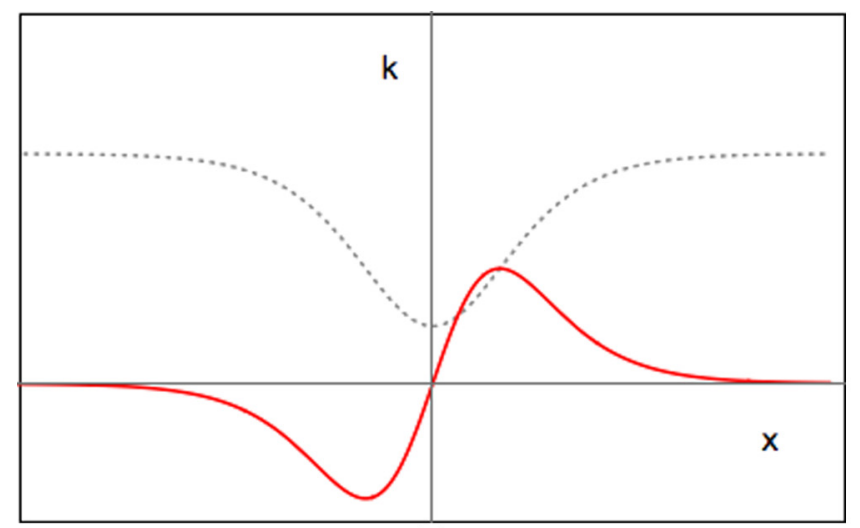

(a)

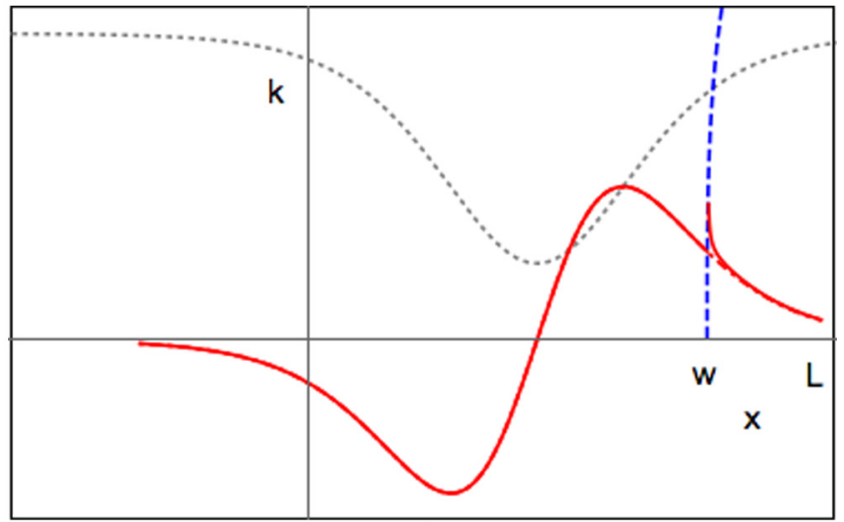

(b)

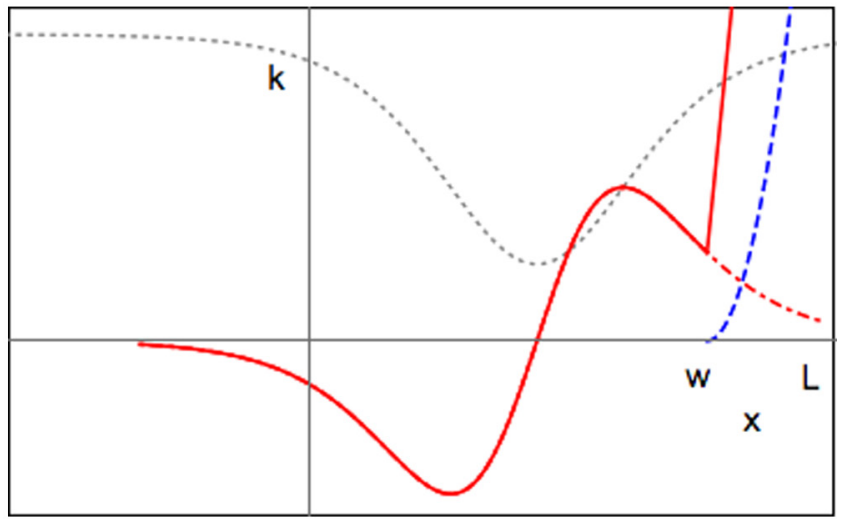

(c)

FIG. 8. The instantaneous wave number K (solid red line) vs $x$ for (a) the central, flat region of the box, and in the proximity of the wall of a power-law box for two values of the exponent: (b) $\alpha=0.001$ and (c) $\alpha=2$. The blue dashed line shows the box potential $V(x)$, which is null in the central region of the condensate $[-w, w]$, and the gray dotted line shows the dark soliton density. The dashed red line shows the wave-number profile $\mathrm{K}(x)$ in the absence of the potential. 
$\partial \phi / \partial x$. It follows that upon entering the wall region, the generation of new wave numbers changes from $\partial|u(x, 0)|^{2} / \partial x$ to $\partial\left[|u(x, 0)|^{2}+V(x)\right] / \partial x$. This immediately suggests that any potential satisfying $\partial[V(x)] / \partial x \neq 0$ is dissipative for the soliton. However, the numerical simulations show nondissipative reflection of the soliton for sufficiently steeps walls for both the power-law box (Fig. 4) and exponential box (Fig. 5). To help understand this, Fig. 8 shows a sketch of the wave numbers $\mathrm{K}=\partial \phi / \partial x$ in the absence of dispersion (solid and dashed red line) for a power-law trap, alongside the dark soliton profile (dotted gray line) and the walls of the box trap (dashed blue line); this is shown for Fig. 8(a) the flat region of the box, and Figs. 8(b) and 8(c) the proximity of the wall for $\alpha=0.001$ and $\alpha=2$, respectively. Note that in the calculation of $\mathrm{K}$, we take $u(x, 0)$ to be the dark soliton (2.4). In Fig. 8(c) that for $x>$ $w$ the wave numbers are skipped by the presence of the wall: The solid red line is quite different from the dashed-red line, corresponding to the absence of the potential. Thus, for $\alpha=2$ [Fig. 8(c)] the potential strongly modifies the instantaneous wave number $\mathrm{K}$, shifting it upwards and supplying energy to some density waves which run away from the soliton [see Figs. 2(d)]. Meanwhile for $\alpha=0.001$ [Fig. 8(b)] the two curves match well, with a small difference only in the region around $x=w$, and the potential wall does not induce any wave emission from the dark soliton. Note that the small discrepancy for $\alpha=0.001$ around $x=w$ arises from the discontinuity in the derivative of $V(x)$ which enters $\mathrm{K}$; in the presence of dispersion, quantum pressure acts to smooth over any sudden spatial variation in $V$ (the healing effect of the condensate) and can be expected to smooth over this discrepancy.

In Figs. 2, 3, and 6, after the dissipative interaction of the soliton box wall, the "new" dark soliton (with lower energy and higher speed) enters again in the region with null potential, where the GP is completely integrable. The important issue here is that GP (2.2) admits the solution (2.4) for any $k$, namely the new dark soliton (just recovered from the side) may propagate undisturbed again in the BEC without any loss and showing its main features, as for instance to keep its identity after a collision with the other waves (in our case, the sound waves).

Over multiple oscillations in the boxlike trap, the energy loss and speed increase of the soliton (which is very small for a single reflection) can become significant (see Fig. 6). With each reflection the condensate becomes increasingly populated with dispersive density waves, which are soon well distributed through the condensate. This procedure lasts until the density depth $k$ of the soliton is comparable to the amplitude of these dispersive waves (see Fig. 6). It is then hard to distinguish the residual dark soliton from the overlapping waves (see the top of Fig. 6), causing two complications. First, the evaluation of the speed or energy of the soliton becomes affected by these waves overlapping the soliton (causing the scattering in the points in Fig. 7). Second, the interaction of the soliton with these dispersive waves cannot be neglected, even though the soliton keeps its identity after the reflections [63]. Indeed, density waves (sound) can supply energy back to some harmonics of the soliton, enhancing its energy.

\section{CONCLUSIONS}

We have studied the propagation of dark solitons in 1D zero-temperature Bose-Einstein condensates confined by boxlike external potentials consisting of a central flat region, where the condensate is practically free, and two soft walls (power law or Gaussian) of variable height and steepness. In the central region the one-dimensional GP is completely integrable, and dark solitons propagate undisturbed. When a soliton meets a side of the trap, depending on the steepness, the soliton may experience total or partial reflection. In the case of partial reflection, small amplitude density waves (sound) are generated and carry energy away from the soliton, and the soliton's speed increases slightly. We map this energy loss as a function of the wall parameters. The reflection is perfect for almost vertical sides. In the dissipative regime and for multiple reflections, the soliton's decay becomes significant. We relate the dissipation to an imbalance between the dispersion term and the combined nonlinear-plus-potential term in the GPE. The condensate becomes increasingly populated by dispersive density waves and when the soliton's depth reaches the level of these waves, its decay stabilizes. Finally, we can conclude that the stability and dynamics of dark solitons in boxlike traps is fundamentally distinct from that in the well-studied case of harmonic potentials, where the soliton is established to propagate with no net dissipation.

Data supporting this publication are openly available under an Open Data Commons Open Database License [64].

\section{ACKNOWLEDGMENTS}

M.S. acknowledge the financial support of the Istituto Nazionale di Alta Matematica (GNFM-Gruppo Nazionale della Fisica Matematica). N.G.P. acknowledges funding from the Engineering and Physical Sciences Research Council (Grant No. EP/M005127/1).
[1] Y. S. Kivshar and B. Luther-Davies, Phys. Rep. 298, 81 (1998).

[2] P. Emplit, J. P. Hamaide, F. Reynaud, C. Froehly, and A. Barthelemy, Opt. Commun. 62, 374 (1987).

[3] D. Krökel, N. J. Halas, G. Giuliani, and D. Grischkowsky, Phys. Rev. Lett. 60, 29 (1988).

[4] A. M. Weiner, J. P. Heritage, R. J. Hawkins, R. N. Thurston, E. M. Kirschner, D. E. Leaird, and W. J. Tomlinson, Phys. Rev. Lett. 61, 2445 (1988).
[5] M. Chen, M. A. Tsankov, J. M. Nash, and C. E. Patton, Phys. Rev. Lett. 70, 1707 (1993).

[6] R. Heidemann, S. Zhdanov, R. Sütterlin, H. M. Thomas, and G. E. Morfill, Phys. Rev. Lett. 102, 135002 (2009).

[7] E. Smirnov, C. E. Rüter, M. Stepić, D. Kip, and V. Shandarov, Phys. Rev. E 74, 065601 (2006).

[8] A. Chabchoub, O. Kimmoun, H. Branger, N. Hoffmann, D. Proment, M. Onorato, and N. Akhmediev, Phys. Rev. Lett. 110, 124101 (2013). 
[9] D. J. Frantzeskakis, J. Phys. A 43, 213001 (2010).

[10] S. Stenholm, Phys. Rep. 363, 173 (2002).

[11] R. Carretero-González, D. J. Frantzeskakis, and P. G. Kevrekidis, Nonlinearity 21, R139(R) (2008).

[12] E. P. Gross, Nuovo Cimento 20, 454 (1961).

[13] E. P. Gross, J. Math. Phys. 4, 195 (1963).

[14] L. P. Pitaevskii, J. Exp. Theor. Phys. 13, 451 (1961).

[15] S. Burger, K. Bongs, S. Dettmer, W. Ertmer, K. Sengstock, A. Sanpera, G. V. Shlyapnikov, and M. Lewenstein, Phys. Rev. Lett. 83, 5198 (1999).

[16] J. Denschlag, J. E. Simsarian, D. L. Feder, C. W. Clark, L. A. Collins, J. Cubizolles, L. Deng, E. W. Hagley, K. Helmerson, W. P. Reinhardt, S. L. Rolston, B. I. Schneider, and W. D. Phillips, Science 287, 97 (2000).

[17] Z. Dutton, M. Budde, C. Slowe, and L. V. Hau, Science 293, 663 (2001).

[18] G.-B. Jo, J.-H. Choi, C. A. Christensen, T. A. Pasquini, Y.-R. Lee, W. Ketterle, and D. E. Pritchard, Phys. Rev. Lett. 98, 180401 (2007).

[19] P. Engels and C. Atherton, Phys. Rev. Lett. 99, 160405 (2007).

[20] C. Becker, S. Stellmer, P. S.-Panahi, S. Dörscher, M. Baumert, E.-M. Richter, J. Kronjäger, K. Bongs, and K. Sengstock, Nat. Phys. 4, 496 (2008).

[21] J. J. Chang, P. Engels, and M. A. Hoefer, Phys. Rev. Lett. 101, 170404 (2008).

[22] S. Stellmer, C. Becker, P. Soltan-Panahi, E.-M. Richter, S. Dörscher, M. Baumert, J. Kronjäger, K. Bongs, and K. Sengstock, Phys. Rev. Lett. 101, 120406 (2008).

[23] A. Weller, J. P. Ronzheimer, C. Gross, J. Esteve, M. K. Oberthaler, D. J. Frantzeskakis, G. Theocharis, and P. G. Kevrekidis, Phys. Rev. Lett. 101, 130401 (2008).

[24] L. M. Aycock, H. M. Hurst, D. Genkina, H.-I. Lu, V. Galitski, and I. B. Spielman, arXiv:1608.03916 (unpublished).

[25] W. P. Reinhardt and C. W. Clark, J. Phys. B 30, L785 (1997).

[26] Th. Busch and J. R. Anglin, Phys. Rev. Lett. 84, 2298 (2000).

[27] G. Huang, J. Szeftel, and S. Zhu, Phys. Rev. A 65, 053605 (2002).

[28] N. G. Parker, N. P. Proukakis, M. Leadbeater, and C. S. Adams, Phys. Rev. Lett. 90, 220401 (2003).

[29] D. E. Pelinovsky, D. J. Frantzeskakis, and P. G. Kevrekidis, Phys. Rev. E 72, 016615 (2005).

[30] N. G. Parker, N. P. Proukakis, and C. S. Adams, Phys. Rev. A 81, 033606 (2010).

[31] D. E. Pelinovsky, Y. S. Kivshar, and V. V. Afanasjev, Phys. Rev. E 54, 2015 (1996).

[32] P. O. Fedichev, A. E. Muryshev, and G. V. Shlyapnikov, Phys. Rev. A 60, 3220 (1999).

[33] A. E. Muryshev, G. V. Shlyapnikov, W. Ertmer, K. Sengstock, and M. Lewenstein, Phys. Rev. Lett. 89, 110401 (2002).

[34] B. Jackson, N. P. Proukakis, and C. F. Barenghi, Phys. Rev. A 75, 051601(R) (2007).

[35] G. Theocharis, P. G. Kevrekidis, M. K. Oberthaler, and D. J. Frantzeskakis, Phys. Rev. A 76, 045601 (2007).
[36] A. E. Muryshev, H. B. van Linden van den Heuvell, and G. V. Shlyapnikov, Phys. Rev. A 60, R2665(R) (1999).

[37] L. D. Carr, M. A. Leung, and W. P. Reinhardt, J. Phys. B: At. Mol. Opt. Phys. 33, 3983 (2000).

[38] D. L. Feder, M. S. Pindzola, L. A. Collins, B. I. Schneider, and C. W. Clark, Phys. Rev. A 62, 053606 (2000).

[39] B. P. Anderson, P. C. Haljan, C. A. Regal, D. L. Feder, L. A. Collins, C. W. Clark, and E. A. Cornell, Phys. Rev. Lett. 86, 2926 (2001).

[40] M. A. Hoefer and B. Ilan, Phys. Rev. A 94, 013609 (2016).

[41] N. G. Parker, N. P. Proukakis, M. Leadbeater, and C. S. Adams, J. Phys. B 36, 2891 (2003).

[42] A. J. Allen, D. P. Jackson, C. F. Barenghi, and N. P. Proukakis, Phys. Rev. A 83, 013613 (2011).

[43] P. G. Kevrekidis, R. Carretero-Gonzalez, G. Theocharis, D. J. Frantzeskakis, and B. A. Malomed, Phys. Rev. A 68, 035602 (2003).

[44] N. G. Parker et al., J. Phys. B 37, S175 (2004).

[45] D. J. Frantzeskakis, G. Theocharis, F. K. Diakonos, P. Schmelcher, and Y. S. Kivshar, Phys. Rev. A 66, 053608 (2002).

[46] N. P. Proukakis, N. G. Parker, D. J. Frantzeskakis, and C. S. Adams, J. Opt. B: Quantum Semiclass. Opt. 6, S380 (2004).

[47] A. Radouani, Phys. Rev. A 68, 043620 (2003).

[48] A. Radouani, Phys. Rev. A 70, 013602 (2004).

[49] N. Bilas and N. Pavloff, Phys. Rev. A 72, 033618 (2005).

[50] I. Hans, J. Stockhofe, and P. Schmelcher, Phys. Rev. A 92, 013627 (2015).

[51] N. Bilas and N. Pavloff, Phys. Rev. Lett. 95, 130403 (2005).

[52] T. P. Meyrath, F. Schreck, J. L. Hanssen, C.-S. Chuu, and M. G. Raizen, Phys. Rev. A 71, 041604(R) (2005).

[53] J. J. P. van Es, P. Wicke, A. H. van Amerongen, C. Rétif, S. Whitlock, and N. J. van Druten, J. Phys. B 43, 155002 (2010).

[54] L. Chomaz, L. Corman, T. Bienaime, R. Desbuquois, C. Weitenberg, S. Nascimbene, J. Beugnon, and J. Dalibard, Nat. Commun. 6, 6162 (2015).

[55] A. L. Gaunt, T. F. Schmidutz, I. Gotlibovych, R. P. Smith, and Z. Hadzibabic, Phys. Rev. Lett. 110, 200406 (2013).

[56] F. Dalfovo, S. Giorgini, L. P. Pitaevskii, and S. Stringari, Rev. Mod. Phys. 71, 463 (1999).

[57] D. S. Petrov, G. V. Shlyapnikov, and J. T. M. Walraven, Phys. Rev. Lett. 85, 3745 (2000).

[58] A. Görlitz et al., Phys. Rev. Lett. 87, 130402 (2001).

[59] T. Brugarino and M. Sciacca, J. Math. Phys. 51, 093503 (2010).

[60] J. Brand and W. P. Reinhardt, Phys. Rev. A 65, 043612 (2002).

[61] S. Komineas and N. Papanicolaou, Phys. Rev. A 68, 043617 (2003).

[62] C. F. Barenghi and N. G. Parker, A Primer on Quantum Fluids (Springer, Berlin, 2016).

[63] I. Oreshnikov, R. Driben, and A. V. Yulin, Opt. Lett. 40, 4871 (2015).

[64] N. Parker, C. Barenghi, and M. Sciacca, Newcastle University Data (2016), doi: 10.17634/101785-4. 